\title{
The use of a propeller flap raised on a previous injured and skin-grafted lower leg area to cover exposed tibia
}

\section{Correspondence}

Nefer Fallico, M.D. Via Val Savio 3, 00141, Rome, Italy.

Email: nefer.fallico@gmail.com

In lower limb reconstruction, defects of the lower third of the leg are a challenging problem, due to the paucity of local tissues available for reconstruction. Although free flaps are considered the gold standard for such defects (Hallock, Wei, \& Mardini, 2006; Kang, Chung, Chang, \& Kim, 2013; Ponten, 1981), propeller perforator flaps are an alternative option for patients with comorbidities that may contraindicate a free tissue transfer or with poor local conditions (Karki, \& Narayan, 2012; Teo, 2010).

We report our experience with a propeller flap raised on a previous injured and skin grafted lower leg area in which the muscular fascia was preserved.

We report a 39-year-old lady who had had a lower leg degloving and open fracture stabilized with an external-fixator in Nigeria 2 months previously, having been run over by a trailer (Figure 1A). Although there had been extensively skin grafted, bone remained exposed. A free flap was not suitable as the patient was a hemophiliac and only the posterior tibial artery remained. Although amputation was discussed, the patient was unwilling to consider it. So we designed a propeller flap based on a posterior tibial perforator in a previously skingrafted area in which the muscular fascia was preserved (Figure 1B). The donor site is grafted with a $10 \times 6$ meshed partial thickness skin graft from the right tight (Figure 1C). The flap survived and the patient was successfully discharged (Figure 1D).

Over the years, propeller flaps have been found to be a reliable source for covering limbs defects, allowing surgeons to avoid the more expensive and complicated microsurgical procedures.

Provided that the deep fascial system is undamaged, coverage of exposed fracture in the lower leg can be carried out by using a propeller flap even in traumatized and previously skin grafted limbs. In this case, the perforator that remained intact going into the muscular fascia, must also have sufficiently vascularized the overlying skin graft.

This report shows that even in a severely traumatized lower limb, which has had an extensive degloving and skin grafting, a perforator

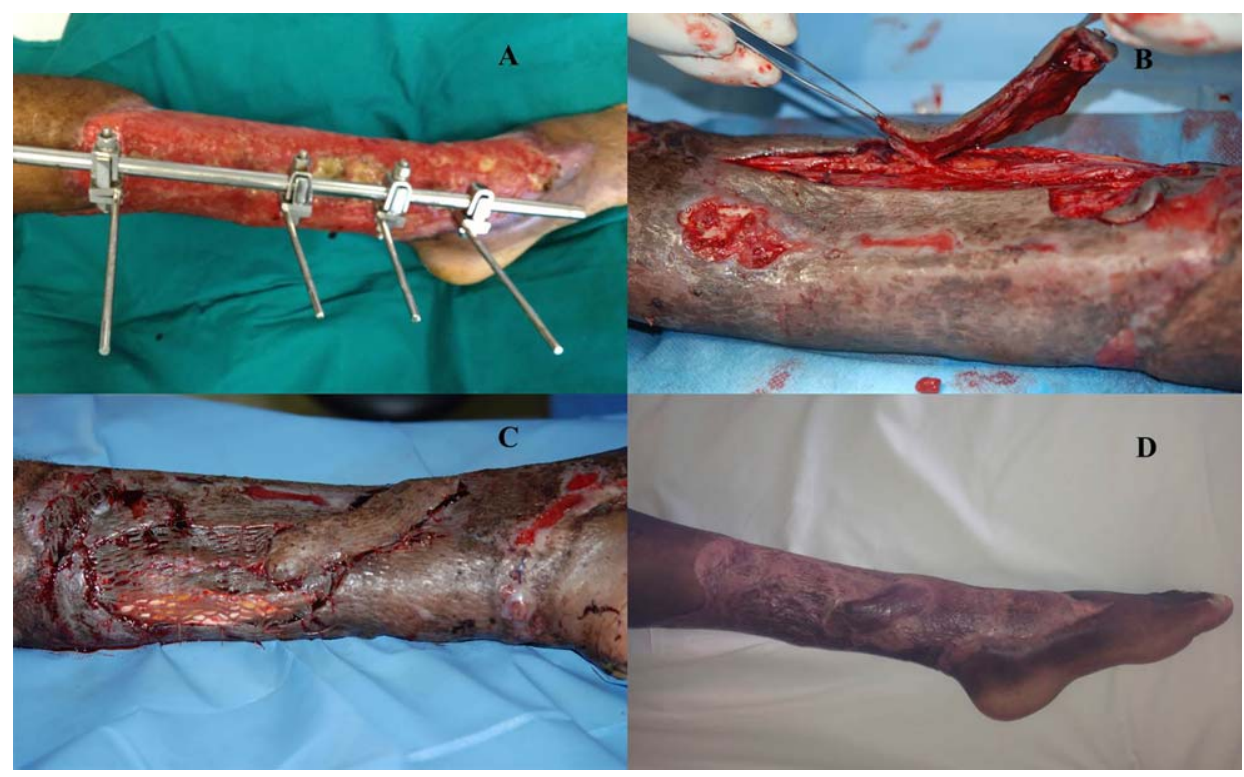

FIGURE 1 A: Complete degloving of the left leg and exposed tibial fracture stabilized by external fixators. B: A $10 \mathrm{~cm} \times 15 \mathrm{~cm}$ propeller flap based on two perforators from the distal posterior tibial artery is harvested on a previous skin-grafted area. The propeller itself consisted of muscular fascia and the graft coated onto it. C: Immediate postoperative aspect. D: Six-month postoperative aspect 
flap is possible and represents a good salvage option in the patient who has co-morbidities that preclude a free flap reconstruction.

\section{CONFLICT OF INTEREST}

The authos declare that they have no conflict of interest.

Luca Andrea Dessy, MD, PhD, DEBOPRAS

Nefer Fallico, MD

Ilaria Casula, MD

Diego Ribuffo, MD, PhD

Department of Plastic and Reconstructive Surgery

Viale del Policlinico 151

"Sapienza" University of Rome

Rome, 00161, Italy

\section{REFERENCES}

Hallock, G. G., Wei, F. C., \& Mardini, S. (2006). Lower extremity reconstruction. In: P. Blondeel, S. F. Morris, \& G. G. Hallock (Eds.), Perforator flaps: Anatomy, technique and clinical applications (pp. 795-817). St. Louis: QMP Publishing.

Kang, M. J., Chung, C. H., Chang, Y. J., \& Kim, K. H. (2013). Reconstruction of the lower extremity using free flaps. Archives of Plastic Surgery, 40, 575-583.

Karki, D., \& Narayan, R. P. (2012). The versatility of perforator-based propeller flap for reconstruction of distal leg and ankle defects. Plastic Surgery International, 2012, 303247.

Ponten, B. (1981). The fasciocutaneus flap: Its use in soft tissue defects of lower leg. British Journal of Plastic Surgery, 34, 215220.

Teo, T. C. (2010). The propeller flap concept. Clinics in Plastic Surgery, 37, 615-626. 